\title{
Carnets
}

Revue électronique d'études françaises de l'APEF

Deuxième série - 6 | 2016

Exotopies de Barthes

\section{Après quoi rien à dire}

Délibération de Barthes et réponse de Kafka

\section{Emmanuel Bouju}

\section{(2) OpenEdition \\ Journals}

Édition électronique

URL : http://journals.openedition.org/carnets/840

DOI : $10.4000 /$ carnets. 840

ISSN : 1646-7698

Éditeur

APEF

Référence électronique

Emmanuel Bouju, «Après quoi rien à dire », Carnets [En ligne], Deuxième série - 6 | 2016, mis en ligne le 31 janvier 2016, consulté le 19 avril 2019. URL : http://journals.openedition.org/carnets/840 ; DOI :

$10.4000 /$ carnets. 840

Ce document a été généré automatiquement le 19 avril 2019

\section{(c) (i) \&}

Carnets est mis à disposition selon les termes de la licence Creative Commons - Atribution - Pas d'utilisation commerciale 4.0 International. 


\title{
Après quoi rien à dire
}

\author{
Délibération de Barthes et réponse de Kafka
}

\section{Emmanuel Bouju}

« De qui suis-je le contemporain?»

(Comment vivre ensemble, Roland Barthes)

1 Lire Barthes, c'est déchirant - à la façon dont Barthes utilisait le qualificatif dans "Délibération", l'un de ses tout derniers textes: «En pratiquant à outrance une forme désuète d'écriture, est-ce que je ne dis pas que j'aime la littérature, que je l'aime d'une façon déchirante, au moment même où elle dépérit?» (Barthes, 2002a : 680) Lire Barthes aujourd'hui, c'est retrouver, dans l'impossible désuétude de son écriture, l'actualité vive d'une interrogation, que nous tous posons à travers lui : comment dire que nous aimons la littérature, sans craindre, à notre tour, qu'elle ne dépérisse (ou qu'elle n'ait déjà dépéri) ? En «pratiquant à outrance » la lecture de Barthes, est-ce que nous ne disons pas que nous aimons la littérature jusqu'au déchirement?

2 Lire Barthes, un siècle après sa naissance, c'est déchirant, ou pour le dire de façon plus énergique et autrement désuète, on pourrait dire : lire Barthes, ça déchire.

3 Cela point et déchire, dans la coïncidence entre "ça a été » et «c'est», dans la contemporanéité constante de Barthes - « survivant » à lui-même comme Kafka le disait, dans son Journal, de l'écrivain capable de « voir sous les décombres »1.

4 Car nous tous qui le lisons aujourd'hui - tels ses disciples ou, c'est selon, tels les médecins de Molière penchés au-dessus du «faux moribond » (selon la comparaison ironique que fit le jeune Barthes en commentant en 1943 le numéro de Confluences sur « les problèmes du Roman ») -, nous sommes tous ses contemporains. Ou du moins nous sommes tous contemporains d'un Barthes, du Barthes que nous préférons.

5 Car si Barthes déchire, c'est aussi parce qu'il est multiple: Barthes est "un multiple » comme on dirait en art, et aussi «un multiple de lui-même» comme on dirait en mathématiques, à l'instar de ce «Barthes puissance 3 » suscité, il y a quarante ans, par Maurice Nadeau pour commenter Roland Barthes par Roland Barthes dans La Quinzaine littéraire ( $1^{\mathrm{er}}$ mars 1975) - un Barthes puissance 3 qui ressemblerait assez à cette image de Pessoa: 


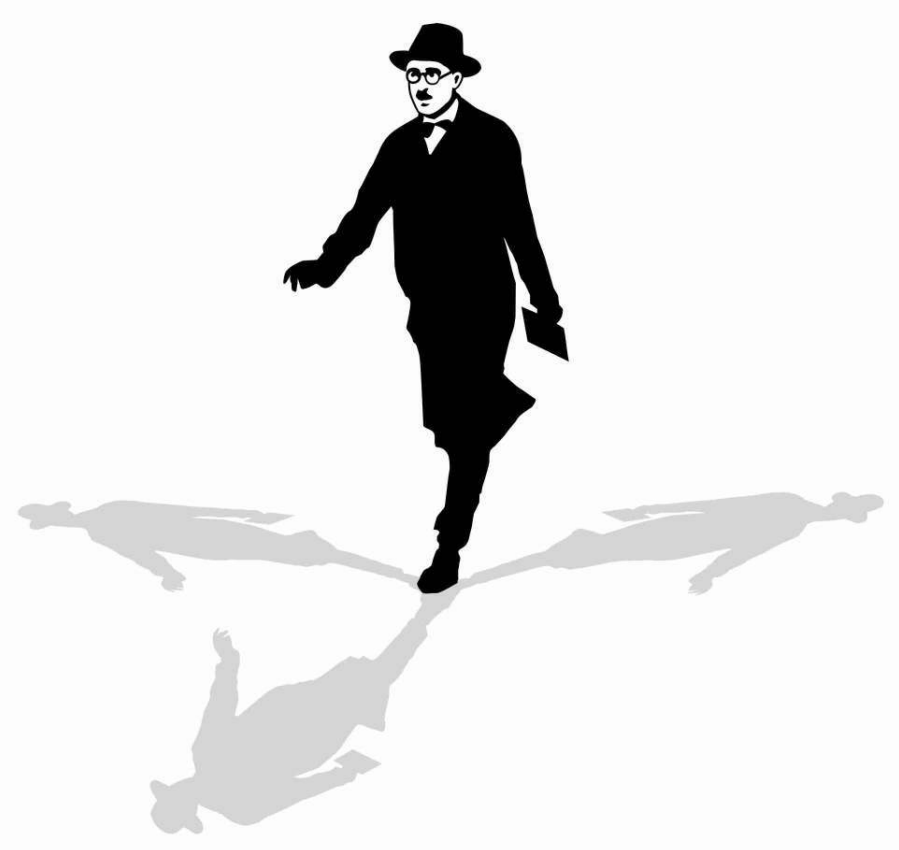

6 Susan Sontag disait bien de lui, à la manière pessoenne, qu'il était le « berger du troupeau de lui-même", dans son hommage posthume de 1981, "Je me souviens de Roland Barthes » (Sontag, 2013 : 205-218). Que Barthes soit multiple et un à la fois, c'est certes un topos de la critique - comme le rappelle à juste titre Tiphaine Samoyault dans sa très belle biographie (Samoyault, 2015) - mais c'est aussi le fait d'une multiplicité inscrite dans son nom - ce qu'il signale à sa façon dans ce même « Barthes puissance 3 ", en faisant (comme Pessoa) de «son propre nom», de «son Nom Propre», «le plus éprouvé des pseudonymes» (Barthes, 2002a: 777) : car ce $s$ muet qui clôt le nom propre (ou le pseudonyme, ou l'orthonyme) de Barthes ${ }^{2}$ - et que l'on entend seulement dans l'adjectivation antonomastique du barthésien, là où le $\mathrm{S}$ résonne, forcément, comme un $\mathrm{Z}$ -, ce $s$ muet dans «Barthes» s'apparenterait assez, orthographiquement et phonétiquement, à une marque du pluriel ${ }^{3}$.

7 Barthes est pluriel, donc, et chacun, maître ès Barthes, aime à invoquer, imaginer, inventer le sien. « Lequel est le vrai ? » comme disait Antoine Compagnon.

Barthes a indirectement répondu à cette question dans son très bel essai critique de « La réponse de Kafka » (1960), en écrivant: " la seule tâche de l'artiste, c'est d'explorer des significations possibles, dont chacune prise à part ne sera que mensonge (nécessaire) mais dont la multiplicité sera la vérité même de l'écrivain. » (Barthes, 1960 : 143-147).

9 Et ainsi, en remontant de « Délibération » (où apparaît le Journal de Kafka), à « La réponse de Kafka » (écrit en marge du Kafka de Marthe Robert), je voudrais pour ma part invoquer ce Barthes dont le dialogue, assez discret, assez secret avec Kafka a été pour lui l'une des occasions d'approcher « la vérité de l'écrivain ». 


\title{
Oui, mais (encore)
}

10 Le Barthes de «la réponse de Kafka », en 1960, c'est celui de la lecture et de l'écoute de Marthe Robert: un Barthes pré-kristévien si l'on peut dire, puisque le premier séminaire auquel Kristeva assiste début 1966 est précisément un séminaire dans lequel intervient encore Marthe Robert ; une préfiguration du « dernier » Barthes aussi en un sens, puisque par le hasard de l'accident final, on peut penser que l'une des dernières lettres que Barthes ait reçues était de la main même de Marthe Robert, et surtout parce qu’à «la réponse de Kafka » Barthes lui-même a répliqué, en écho, en faisant du dernier texte livré à Tel Quel, «Délibération » déjà cité, un texte portant sur l'écriture du Journal au miroir de celui de Kafka (Barthes y «copie » même littéralement Kafka dans les extraits qu'il livre de son Journal, comme le souligne à juste titre Tiphaine Samoyault).

11 Dans ces deux textes, séparés donc par toute une œuvre immense (symptôme de la multiplicité barthésienne), revient, étonnamment, une même expression, empruntée à Kafka via Marthe Robert : le « oui, mais » de la réponse de Kafka au monde :

Marthe Robert dit excellemment que les rapports de Kafka et du monde sont réglés par un perpétuel : oui, mais... Au succès près, on peut le dire de toute notre littérature moderne (et c'est en cela que Kafka l'a vraiment fondée), puisqu'elle confond d'une façon inimitable le projet réaliste (oui au monde) et le projet éthique (mais...) (Barthes, $1960: 145)$.

Comme le pervers (dit-on), assujetti au oui, mais, je sais que mon texte est vain, mais en même temps (d'un même mouvement) je ne puis m'arracher à la croyance qu'il existe. (Barthes, 2002a : 678)

Ce « oui mais » est une trouvaille extraordinaire, que Barthes étend à l'origine, d'un seul geste, à toute la littérature "moderne » - avant que de le reprendre pour lui-même, sous le signe, néo-kafkaïen, de la "perversion » (terme clinique et pas forcément péjoratif, étant donné l'usage qu'en a souvent fait Barthes pour lui-même et pour la littérature ${ }^{4}$ ) : cette croyance malgré le savoir, cette perversion littérale de la conscience de la vanité qui fait d'elle la puissance active du déchirement, c'est une force qui emplit les essais critiques et éclaire jusqu'à la littérature d'aujourd'hui :

\begin{abstract}
Le trajet qui sépare le oui du mais, c'est toute l'incertitude des signes, et c'est parce que les signes sont incertains qu'il y a une littérature. La technique de Kafka dit que le sens du monde n'est pas énonçable, que la seule tâche de l'artiste, c'est d'explorer des significations possibles, dont chacune prise à part ne sera que mensonge (nécessaire) mais dont la multiplicité sera la vérité même de l'écrivain. Voilà le paradoxe de Kafka : l'art dépend de la vérité, mais la vérité, étant indivisible, ne peut se connaître elle-même: dire la vérité, c'est mentir. Ainsi l'écrivain est la vérité, et pourtant quand il parle, il ment : l'autorité d'une œuvre ne se situe jamais au niveau de son esthétique, mais seulement au niveau de l'expérience morale qui en fait un mensonge assumé ; ou plutôt, comme dit Kafka corrigeant Kierkegaard : on ne parvient à la jouissance esthétique de l'être qu'à travers une expérience morale et sans orgueil (Barthes, $1960: 146)$
\end{abstract}

De façon magistrale, et en concluant à la Borges (lequel faisait justement de Kierkegaard un "précurseur» de Kafka), Barthes associe dans cet article incertitude des signes, expérience morale et technique de l'écriture, et prépare en amont, par la métaphore de l'homéostat d'Ashby (ancêtre de la cybernétique), la critique ultérieure de l'autorité (ici encore rattachée à la vérité de l'écrivain). 
littérature contemporaine - valeurs en apparence négatives mais d'une productivité immense encore aujourd'hui : «l'instabilité du jugement » et le « défaut du sujet »; le fait de «ne répondre à aucune mission » et d'offrir l'horizon d'un texte-Album dont les feuillets «sont non seulement permutables, mais surtout suppressibles à l'infini» (comme dans l'actuelle configuration en hypertexte numérique, comme le remarque à juste titre Tiphaine Samoyault); l'écriture comme «copie de copie» et surtout l'incapacité de prouver, «non seulement ce qu'elle dit mais encore qu'il vaut la peine de le dire » (Barthes, 2002b : 678-680).

19 Ainsi par exemple le brillant romancier et critique anglais Adam Thirlwell, qui prend Barthes pour héros de sa quête du "Livre multiple », en vient-il à faire (sérieusement et ironiquement tout à la fois) de $L a$ Préparation du roman, non pas tant la préparation du roman barthésien (qu'on l'ait crue impossible ou non), que la préparation, sinon de tout le roman contemporain, du moins de celui que Thirlwell appelle de ses vœux : lancé en quête de ces «moments de vérité ", de "littéralité totale » dont témoignent la mort du Prince Bolkonski chez Tolstoï et celle de la grand-mère du narrateur de La Recherche, Barthes (ce héros) livre à Thirlwell une formule magique qui décide de sa vocation pour partir à son tour en quête de tels moments, lesquels surgissent, dit Barthes, "de l'ininterprétable, du dernier degré de sens, de l'après quoi plus rien à dire... » : «C'est là, je crois, que Barthes formule en quelque sorte une proposition pour une future avant-garde du roman: (...) une histoire peut devenir la preuve de choses qu'on ne pourra jamais logiquement prouver $»^{5}$ (Thirlwell, $2012: 37$ ).

20

À ce titre, la « Leçon inaugurale » du Collège de France semble encore placée, bien qu'en de tout autres termes, sous cette perspective rhétorique paradoxale du « oui, mais »:

Je disais à l'instant, à propos du savoir, que la littérature est catégoriquement réaliste, en ce qu'elle n'a jamais que le réel pour objet de désir; et je dirai familière, qu'elle est tout aussi obstinément : irréaliste ; elle croit sensé le désir de l'impossible. (Barthes, 2002b : 436)

hétéronymie des choses ", qui "permet d'entendre la langue hors-pouvoir " selon les termes de la Leçon (Barthes, 2002b: 433), découle ainsi directement de cette responsabilité de la forme inscrite, depuis la « réponse de Kafka », en son sein, par le biais du oui, mais.

Or personnellement, je ne crois pas cette leçon définitivement perdue. Et « la réponse de Kafka », comme la "Délibération " barthésienne, sont des textes séminaux pour la littérature contemporaine.

l'allusion, la précision de l'écriture qui « engage » l'écrivain « dans le monde », sont des avancées dont chacun reconnaît la portée (bien que certains fassent mine de les réinventer aujourd'hui) : beaucoup en reconnaissent la portée, non seulement en France (de Simon à Senges), mais aussi partout hors de France, comme chez Zadie Smith, László Krasznahorkai, ou comme chez Gonçalo Tavares (auteur d'un Roland Barthes e Robert Musil) pour prendre un exemple portugais.

De fait, une formule frappante résonne au cœur de la délibération barthésienne : la littérature est sans preuves. Dans cette formule (ce mana?), il faut entendre ce que fait seulement apparaître l'écriture - et la lecture (toujours) avec elle : ce $s$ surnuméraire (à 
quoi prédisposait le nom de Barthes), cette discrète infraction grammaticale (puisqu'on devrait avoir « la littérature est sans preuve » au singulier) qui rappelle celle que Perec pratiqua très volontairement en écrivant « je n'ai pas de souvenirs d'enfance ». De même que chez Perec l'absence des souvenirs d'enfance fait de l'écriture le souvenir de l'enfance, de même ici, c'est l'absence des preuves qui est la preuve même de la littérature. Moimême, répondant pour un numéro récent de la NRF à la question « Que peut (encore) la littérature ?» (Bouju, 2014) (50 ans après le débat sartrien), j'ai cru nécessaire de suivre cette suggestion, en même temps que j'ai suivi celle de Foucault, parente de la « réponse de Kafka» (et exactement contemporaine de la conférence de Barthes à Lisbonne sur « littérature et signification») :

Je crois qu'on pourrait dire ceci, qu'au fond, nous ne croyons plus de nos jours à la liberté politique, et puis le rêve, le fameux rêve d'un homme désaliéné est tombé maintenant dans la dérision. De tant de chimères, que nous est-il resté ? Eh bien, la cendre de quelques mots. Et notre possible, à nous autres hommes d'aujourd'hui, notre possible, nous ne le confions plus aux choses, aux hommes, à l'Histoire, aux institutions, nous le confions aux signes. (Foucault, 2013: 54)

Et ainsi, à la question « que peut (encore) la littérature ? ", j’ai répondu d'abord : « Rien », puis je me suis employé à retourner ce rien, cette absence de preuves, cet impouvoir de la littérature en une puissance nouvelle (de résurrection ironique de l'autorité-fantôme, de contra-diction des discours de pouvoir et d'écriture alter-historiographique - chez Elfriede Jelinek, Roberto Bolaño, Imre Kertész et bien d'autres) : placée sous le signe barthésien du Oui, mais (encore), la (meilleure) littérature contemporaine allie forme et éthique en confiant le possible au signe, par le geste du renversement du rien en puissance substantive (Rien).

Geste classique - ruse odysséenne - d'une puissance d'inversion (ou de perversion) propre à l'écriture et à sa lecture : comme chez Paul Celan - dont le nom de plume inverse le nom propre (Ancel) - et qui fait, dans le poème Psalm (Psaume), du niemand adverbial (personne) une puissance nouvelle en le substantivant par une simple majuscule ( Niemand, Personne), afin de rendre possible de nouveau « un Rien », la « rose de Rien, la rose de Personne » :

\begin{tabular}{|l|l|}
\hline $\begin{array}{l}\text { Niemand knetet uns wieder aus Erde und } \\
\text { Lehm } \\
\text { niemand bespricht unserem Staub. } \\
\text { Niemand. }\end{array}$ & $\begin{array}{l}\text { Personne ne nous pétrira de nouveau dans la terre et } \\
\text { l'argile, } \\
\text { personne ne soufflera la parole sur notre poussière. } \\
\text { Personne. }\end{array}$ \\
\hline $\begin{array}{l}\text { Gelobt seist du, Niemand. } \\
\text { Dir zu lieb wollen } \\
\text { wir blühn. } \\
\text { Dir entgegen. }\end{array}$ & $\begin{array}{l}\text { Loué sois-tu, Personne. } \\
\text { C'est pour toi que nous voulons } \\
\text { fleurir } \\
\text { A ta rencontre. }\end{array}$ \\
\hline $\begin{array}{l}\text { Ein Nichts } \\
\text { waren wir, sind wir, werden } \\
\text { wir bleiben, blühend: } \\
\text { die Nichts -, die }\end{array}$ & $\begin{array}{l}\text { Un Rien, } \\
\text { voilà ce que nous fûmes, sommes et } \\
\text { resterons, fleurissant : } \\
\text { la rose de Rien, la } \\
\text { rose de Personne. }\end{array}$ \\
\hline
\end{tabular}




\begin{tabular}{|l|l|}
\hline & Un Rien, \\
& voilà ce que nous fûmes, sommes et \\
resterons, fleurissant: \\
la rose de Rien, la \\
rose de Personne
\end{tabular}

Ou comme chez Emily Dickinson avant lui (\# 1563) qui écrivait :

\begin{tabular}{|l|l|}
\hline By homely gift and hindered words & Par don modeste et Mots entravés \\
The human heart is told & Le cœur humain (n')apprend \\
Of Nothing - & Rien - \\
'Nothing' is the force & 'Rien' est la force \\
That renovates the World - & Qui rend le Monde neuf - \\
\hline
\end{tabular}

24 Geste classique donc, mais aussi geste éminemment barthésien, que ce geste $d u$ «retournement » : peut-être inspiré du compagnonnage avec Flaubert et son utopie du « livre sur rien »; peut-être inspiré de Brecht, qui annonçait déjà : « après nous viendra : rien qui ait un nom »- en prémisse de la mort de l'auteur, et en tant que l'auteur porte désormais le nom de rien ni de personne, comme Pessoa ${ }^{6}$. Ou, plus explicitement chez Barthes, geste inspiré du Cuisinier d'Arcimboldo : ce tableau réversible qu'il évoque dans "Arcimboldo ou Rhétoriqueur et magicien ${ }^{7}$ - en précisant que pour inverser la signification, il suffit que « la lecture tourne, sans cran d'arrêt » (Barthes 2002b : 495) (la même image étant reprise dans la Leçon, et dans nombre d'autres lieux rhétoriques de l'œuvre de Barthes).

Or c'est exactement ce même principe de retournement (sans cran d'arrêt) que l'on retrouve au cœur du raisonnement de "Délibération»: oui, «la littérature est sans preuves ", mais encore, «à ce point, tout se retourne, car de son impuissance à la preuve, qui l'exclut du ciel serein de la Logique, le Texte tire une souplesse, qui est comme son essence, ce qu'il possède en propre. » (Barthes, 2002b : 681).

Et Barthes, à ce moment précis, comme pour apporter la preuve de cette absence de preuves, de citer Kafka ${ }^{8}$ :

Kafka [écrit donc Barthes, en suscitant son fantôme] - dont le Journal est peut-être le seul qui puisse être lu sans aucune irritation - dit à merveille cette double postulation de la littérature, la Justesse et l'Inanité : '(...) J'examinais les souhaits que je formais pour la vie. Celui qui se révéla le plus important ou le plus attachant fut le désir d'acquérir une façon de voir la vie (et, ce qui était lié, de pouvoir par écrit en convaincre les autres) dans laquelle la vie conserverait son lourd mouvement de chute et de montée, oui, mais serait reconnue en même temps, et avec une clarté non moins grande, pour un rien, un rêve, un état de flottement'.

À Barthes qui, dans ses derniers textes, ne cesse de délibérer (« l'air de rien » dit Tiphaine Samoyault) sur la justesse et l'inanité de toute son entreprise sous les termes du «Quoi, tout cela pour rien ? $»^{10}$, Kafka donne sa réponse, en forme de clé d'écriture : tout cela pour un rien (un rêve, un état de flottement). Et ainsi «être pour rien", "faire sens avec rien ", toutes expressions barthésiennes ${ }^{11}$ (Samoyault, $2015: 264$ et 374), s'entendent de façon positive, et deviennent la clé d'une reconquête de la littérature et de la signification, tout ensemble. 
Aussi, après la réponse de Kafka et la délibération barthésienne, et 100 ans exactement après que cette note de Kafka sur sa « façon de voir la vie » a coïncidé avec la naissance de Barthes, dans "l'après quoi Rien à dire ", entendra-t-on encore la formule, le mana ou la leçon même de l'écriture.

\section{BIBLIOGRAPHIE}

BARTHES, Roland (1960). « La Réponse de Kafka », in Essai critique. Paris : Seuil, « Points ».

BARTHES, Roland (2002a). CEuvres complètes IV, Paris : Seuil.

BARTHES, Roland (2002b). Cuures complètes V, Paris : Seuil.

Bouju, Emmanuel (2014). « Oui, mais (encore). Puissance du roman contemporain », in La Nouvelle

Revue française, no 609 ("Que peut (encore) la littérature? »), sous la direction de Stéphane

AUDEGUY et Philippe FOREST, Paris : Gallimard, 2014, pp. 66-75.

FOUCAULT, Michel (2013). «Le Langage en folie » [émission de l'ORTF, 1963], in La Grand étrange.

Paris : Édtion de l’EHESS, « Audiographie ».

KAFKA, Franz (1921). Journal. 19 octobre 1921.

SAMOYAULT, Tiphaine (2015). Roland Barthes. Biographie, Paris : Seuil.

SONTAG, Susan (2013). «Je me souviens de Roland Barthes », in Sous Le Signe de Saturne. Cuvres

complètes IX. S. 1. : Éditions Bourgois, « Titres 166 », pp. 205-218.

THIRLWELL, Adam (2013). Le Multiple [Miss Herbert, Vintage, 2012], trad. Anne-Laure Tissot. S. 1. :

Éditions de l'olivier.

\section{NOTES}

1. «Celui qui, vivant, ne vient pas au bout de la vie, a besoin d'une main pour écarter un peu le désespoir que lui cause son destin (...), mais de l'autre main peut écrire ce qu'il voit sous les décombres, car il voit autrement et plus de choses que les autres, n'est-il pas mort de son vivant, n'est-il pas l'authentique survivant?» (Franz Kafka, 1921).

2. Tiphaine Samoyault évoque même "le tétrasyllabe Barthes et Foucault », ce qui amuït le s y compris en niant la possibilité d'une liaison (Samoyault, 2015 : 595).

3. Sarkozy lui-même (ce diafoirus) a d'ailleurs cru utile de signaler, dans un de ses discours, que le $s$ de Barthes n'était pas en vérité une marque du pluriel, en prononçant Barthès (comme il aurait pu dire Princesse de Clèvès s'il ne la connaissait si bien) ; ce faisant, on a cru qu'il confondait Barthes avec le goal de l'équipe de France, Barthez... mais on peut aussi penser que par-là, glissant du $z$ au $s$, il entendait copier secrètement et savamment - par quelque Sarkosine de son fait - la Sarrasine de Balzac revue par Barthes.

4. Cf. Nadeau vs Barthes au sujet de la « perversion » de la littérature, dans le dialogue du 13 mars 1974 (repris dans Sur la littérature, PUG, 1980, et cité in Tiphaine Samoyault, 2015 : 217). 
5. Ceci est une formule très importante pour tout ce qui concerne l'écriture actuelle de l'histoire, où la fiction joue souvent le pari d'une autorité sans preuve, sous la forme de ce que j'ai appelé « roman istorique » sans $h$.

6. Tout à fait comme Pessoa - dont le nom est (littéralement) Personne et qui peut, n'étant rien, « être rien, simple figure de roman (...) Une ombre sur un sol irréel, un rêve dans un rêve ».

7. Roland Barthes, «Arcimboldo ou Rhétoriqueur et magicien » (Arcimboldo, F.M.R., 1978), in Barthes (2002b : 493-511).

8. Kafka, dont le Journal porte au demeurant souvent la simple mention « rien » - ce qui est déjà quelque chose - et qui parfois transforme même ce "rien écrire », ou ce "rien écrit », par la formule : « Rien, rien. C'est ainsi que je me suscite des fantômes » (10 mars 2012).

9. Roland Barthes, « Délibération » (2002a: 681). C'est moi qui souligne.

10. BNF, NAF 28630, « Grand fichier », 14 octobre 1979 ; cité par Tiphaine Samoyault (2015: 85).

11. Tiphaine Samoyault (2015: 264 et 379$)$.

\section{RÉSUMÉS}

En remontant le temps de «Délibération » (1979) à «La réponse de Kafka » (1960), cet article examine la façon dont la contemporanéité déchirante de Barthes est liée au dialogue, assez discret, qu'il a entrentenu avec Kafka et qui a été pour lui l'une des occasions d'approcher «la vérité de l'écrivain ».

Rewinding time from « Délibération » (1979) to « La réponse de Kafka » (1960), this article aims to examine the tearing (déchirante) contemporaneity of Barthes through the secret dialogue he had with Kafka: one of his ways to get to « the truth of the writer ».

\section{INDEX}

Mots-clés : Kafka (Franz), vérité, contemporanéité

Keywords : Kafka (Franz), truth, contemporaneity

\section{AUTEUR}

\section{EMMANUEL BOUJU}

Université Rennes 2, Groupe phi

Harvard University

em.bouju@gmail.com 